PROCEEDINGS OF THE

AMERICAN MATHEMATICAL SOCIETY

Volume 125, Number 10, October 1997, Pages 3089-3098

S $0002-9939(97) 04021-5$

\title{
THE AR-PROPERTY FOR ROBERTS' EXAMPLE OF A COMPACT CONVEX SET WITH NO EXTREME POINTS PART 2: APPLICATION TO THE EXAMPLE
}

\author{
NGUYEN TO NHU, JOSE M. R. SANJURJO, AND TRAN VAN AN \\ (Communicated by James West)
}

\begin{abstract}
In this second part of our paper, we apply the result of Part 1 to show that the compact convex set with no extreme points, constructed by Roberts (1977), is an AR.
\end{abstract}

This is the second part of our paper. In this part, we prove that the compact convex set with no extreme points, constructed by Roberts [R1], is an AR.

All notation, used in this part, comes from Part 1.

\section{Roberts' EXAMPLE}

We are going to describe the linear metric space which contains a compact convex set with no extreme points, constructed by Roberts [R1].

Let $\left\{d_{n}\right\}$ be a sequence of natural numbers. Put $m(1)=1$ and inductively define $m(n+1)=d_{n} m(n)$. Let $\pi_{1}=\{[0,1)\}$. Assume that $\pi_{n}$ is a partition of $[0,1)$ into $m(n)$ equal length intervals of the form $[a, b)$. For each $S \in \pi_{n}$, let $\pi_{n+1}(S)$ denote the partition of $S$ into $d_{n}$ equal length subintervals. Then we define the partition $\pi_{n+1}=\cup\left\{\pi_{n+1}(S): S \in \pi_{n}\right\}$ of $[0,1)$ into $m(n+1)$ equal length intervals. Consider the linear space consisting of all functions on $[0,1]$ which are finite linear combinations of characteristic functions of the form $\chi_{[a, b)}$. We denote

$$
E_{n}=\operatorname{span}\left\{\chi_{S}: S \in \pi_{n}\right\} ; E=\bigcup_{n=1}^{\infty} E_{n}
$$

$\left(\chi_{S}\right.$ denotes the characteristic function of $\left.S\right)$;

$$
A_{n}=\left\{m(n) \chi_{S}: S \in \pi_{n}\right\}=\left\{a_{i}^{n}: i=1, \ldots, m(n)\right\} \text { (compare with (10)), }
$$

where $a_{i}^{n}=m(n) \chi_{S_{i}^{n}}, i=1, \ldots, m(n) ; \pi_{n}=\left\{S_{1}^{n}, \ldots, S_{m(n)}^{n}\right\}$. Observe that

$$
E_{1} \subset E_{2} \subset \cdots \subset E \text {, see (30). }
$$

Note that paranorms constructed in [R1] are monotone, norm bounded and total, hence they are monotone $F$-norms. Then "paranorms" can be replaced by

Received by the editors December 17, 1992 and, in revised form, April 1, 1996.

1991 Mathematics Subject Classification. Primary 54C55; Secondary 54D45.

Key words and phrases. Convex set, linear metric space, extreme point, absolute retract.

The first author was supported by the Complutense University of Madrid. 
"monotone $F$-norms". Observe that a paranorm is an $F$-norm if it is norm bounded and total. The following theorem was proved by Roberts [R1, §3].

Theorem 4. For a suitable sequence of natural numbers $\left\{d_{n}\right\}$, there exist sequences of paranorms $\left\{N_{n}\right\}$ and $\left\{N_{n+1}(S): S \in \pi_{n}\right\}$ on $E_{n}$ and on $E_{n+1}(S)$ respectively, such that the following conditions hold:

(i) $N_{1}(x)=\int_{0}^{1}|x(t)| d t$ for every $x \in E_{1}$;

(ii) $N_{n+1}(x)=\inf \left\{N_{n}(y)+\sum\left\{N_{n+1}(S)(x(S)): S \in \pi_{n}\right\}\right\}$ for every $x \in E_{n+1}$, where the infimum is taken over all the expressions of $x$ of the form:

$$
x=y+\sum\left\{x(S): S \in \pi_{n}\right\}, y \in E_{n}, x(S) \in E_{n+1}(S) ;
$$

(iii) $\left\{N_{n}(x)\right\}$ is a decreasing sequence for every $x \in E$ and the formula

$$
N(x)=\lim _{n \rightarrow \infty} N_{n}(x) \text { for } x \in E
$$

defines a monotone $F$-norm of $E$;

(iv) If $x \in E_{n}$ and $N_{n}(x)<4$, then $N_{m}(x)=N_{n}(x)$ for every $m>n$;

(v) The sequence $\left\{A_{n}\right\}$, defined by (31), satisfies the conditions (4) and (5) of Theorem 1, Part 1, where $A_{n+1}(a)=\left\{m(n+1) \chi_{S^{\prime}}: S^{\prime} \in \pi_{n+1}(S)\right\}$ if $a=$ $m(n) \chi_{S} \in A_{n}, S \in \pi_{n}$.

Therefore $C=\overline{\cup_{n=o}^{\infty} \hat{A}_{n}} \subset X$, see (6), is a compact convex set with no extreme points, where $X$ is the completion of $(E, N)$.

Let $\|$.$\| denote the F$-norm on $X$ induced by $N$. Our aim is to show that the compact convex set $C$, defined by Theorem 4, satisfies the conditions of Theorem 2, Part 1. We need the following fact:

Lemma 6. Using the notation (31), for each $i=1, \ldots, m(n)$, if $x \in \operatorname{span} C_{n}\left(a_{i}^{n}\right) \cap$ $E$, see (8), then supp $x \subset S_{i}^{n}$.

Proof. Let $x \in \operatorname{span} C_{n}\left(a_{i}^{n}\right) \cap E$. Take $q>n$ such that $x \in E_{q}$. Then we have

$$
x=\sum_{j=1}^{m(q)} \alpha_{j} a_{j}^{q}, \text { where } a_{j}^{q}=m(q) \chi_{S_{j}^{q}}, j=1, \ldots, m(q) .
$$

Assume on the contrary that $\alpha_{j} \neq 0$ for some $S_{j}^{q} \subset[0,1] \backslash S_{i}^{n}$. We may assume that $j=1$. Take a sequence $\left\{x_{k}\right\} \subset \operatorname{span} \cup_{j=1}^{\infty} \hat{A}_{n+j}\left(a_{i}^{n}\right) \cap E$ such that $x_{k} \rightarrow x$, see (8). Then, we have

$$
\text { supp } x_{k} \subset S_{i}^{n} \text { for every } k \in \mathbb{N} \text {, see (30) and (31). }
$$

For any function $x$ on $[0,1]$ and $A \subset[0,1]$, denote $\left.x\right|_{A}=\chi_{A} x$. Then from (33) we get

$$
x-x_{k}\left|[0,1] \backslash S_{i}^{n}=x\right|[0,1] \backslash S_{i}^{n} \in E_{q} \text { for every } k \in \mathbb{N} .
$$

Since $\left\|x-x_{k}\right\| \rightarrow 0$, by Theorem 4(ii)-(iv), there exists an expression

$$
x-x_{k}=z_{q}(k)+\sum_{j=q}^{n(k)-1} \sum_{i=1}^{m(j)} y_{i}^{j}(k), k \in \mathbb{N},
$$


where $n(k)>q$ is chosen so that $x_{k} \in E_{n(k)}$, and $z_{q}(k) \in E_{q}, y_{i}^{j}(k) \in E_{j+1}\left(S_{i}^{j}\right)$ for $i=1, \ldots, m(j), j=q, \ldots, n(k)-1$, such that

$$
N_{q}\left(z_{q}(k)\right)+\sum_{j=q}^{n(k)-1} \sum_{i=1}^{m(j)} N_{j+1}\left(S_{i}^{j}\right)\left(y_{i}^{j}(k)\right) \rightarrow 0 \text { as } k \rightarrow \infty .
$$

Since $z_{q}(k) \in E_{q}$ we have, see (30) and (31),

$$
z_{q}(k)=\sum_{t=1}^{m(q)} y_{t}(k), \text { where } y_{t}(k)=\lambda_{t}(k) m(q) \chi_{S_{t}^{q}}, t=1, \ldots, m(q) .
$$

From (35) we get $N_{q}\left(z_{q}(k)\right) \rightarrow 0$ as $k \rightarrow \infty$. Since $\left\{z_{q}(k)\right\}$ is a sequence in the finite dimensional space $E_{q}$, equipped with the Hamel base $\left\{\chi_{S_{t}^{q}}: t=1, \ldots, m(q)\right\}$ and the $F$-norm $N_{q}$, it follows from (36) that

$$
N_{q}\left(y_{t}(k)\right) \rightarrow 0 \text { as } k \rightarrow \infty \text {, for every } t=1, \ldots, m(q) .
$$

Observe that for every $j \in\{q, \ldots, n(k)-1\}$ and $i \in\{1, \ldots, m(j)\}$ we have $S_{i}^{j} \subset S_{t}^{q}$ for some $t \in\{1, \ldots, m(q)\}$. Denote

$$
u_{1}(k)=\sum_{S_{i}^{j} \subset S_{1}^{q}} y_{i}^{j}(k) .
$$

Then we have

Claim 6. $y_{1}(k)+u_{1}(k)=\alpha_{1} m(q) \chi_{S_{1}^{q}} \in E_{q}$ for every $k \in \mathbb{N}$.

Proof. Recall that $\left.x\right|_{A}=\chi_{A} x$. Since $S_{1}^{q} \subset[0,1] \backslash S_{i}^{n}$, from (33) and (34) we get

$$
x\left|S_{1}^{q}=x-x_{k}\right| S_{1}^{q}=z_{q}(k)\left|S_{1}^{q}+\sum_{j=q}^{n(k)-1} \sum_{i=1}^{m(j)} y_{i}^{j}(k)\right| S_{1}^{q} .
$$

From (32) and (36) we have

$$
x \mid S_{1}^{q}=\alpha_{1} m(q) \chi_{S_{1}^{q}} \text { and } z_{q}(k) \mid S_{1}^{q}=y_{1}(k) .
$$

Since $y_{i}^{j}(k) \in E_{j+1}\left(S_{i}^{j}\right)$, we get

$$
\sum_{j=q}^{n(k)-1} \sum_{i=1}^{m(j)} y_{i}^{j}(k) \mid S_{1}^{q}=\sum_{S_{i}^{j} \subset S_{1}^{q}} y_{i}^{j}(k)=u_{1}(k), \text { see }(38) .
$$

Consequently, the claim follows from (39).

Since $y_{1}(k) \in E_{q}$, from Claim 6 we get $u_{1}(k) \in E_{q}$ for every $k \in \mathbb{N}$. Therefore, from Theorem 4(ii)-(iv) and from (35) we get

$$
\begin{aligned}
N_{q}\left(u_{1}(k)\right) & \leq \sum_{S_{i}^{j} \subset S_{1}^{q}} N_{j+1}\left(S_{i}^{j}\right)\left(y_{i}^{j}(k)\right) \leq \sum_{t=1}^{m(q)} \sum_{S_{i}^{j} \subset S_{t}^{q}} N_{j+1}\left(S_{i}^{j}\right)\left(y_{i}^{j}(k)\right) \\
& =\sum_{j=q}^{n(k)-1} \sum_{i=1}^{m(j)} N_{j+1}\left(S_{i}^{j}\right)\left(y_{i}^{j}(k)\right) \rightarrow 0 \text { as } k \rightarrow \infty .
\end{aligned}
$$

Hence, from Claim 6 and from (37) we obtain

$$
N_{q}\left(\alpha_{1} m(q) \chi_{S_{1}^{q}}\right) \leq N_{q}\left(y_{1}(k)\right)+N_{q}\left(u_{1}(k)\right) \rightarrow 0 \text { as } k \rightarrow \infty .
$$


Consequently, $N_{q}\left(\alpha_{1} m(q) \chi_{S_{1}^{q}}\right)=0$ and therefore $\alpha_{1}=0$. So, we get a contradiction. The lemma is proved.

\section{The RESUlt}

Now we come to our result in this paper:

Theorem 5. The compact convex set $C$, defined by Theorem 4, is an AR.

Proof. We are going to verify the conditions of Theorem 2. First, observe that Condition (ii) follows from the construction. Let us check Condition (i): if $x_{i} \in$ span $C_{n}\left(a_{i}^{n}\right) \backslash\{\theta\}, i=1, \ldots, m(n)$, then the set $\left\{x_{1}, \ldots, x_{m(n)}\right\}$ is linearly independent in $X$.

Let $x_{i} \in \operatorname{span} C_{n}\left(a_{i}^{n}\right) \backslash\{\theta\}$ and $\lambda_{i} \in \mathbb{R}, i=1, \ldots, m(n)$, such that

$$
\lambda_{1} x_{1}+\cdots+\lambda_{m(n)} x_{m(n)}=\theta \text { where } \lambda_{i} \in \mathbb{R}, i=1, \ldots, m(n) .
$$

We shall prove that $\lambda_{i}=0$ for every $i=1, \ldots, m(n)$. Assume on the contrary that $\lambda_{i} \neq 0$ for some $i \in\{1, \ldots, m(n)\}$. We may assume that $i=1$. Then we get

$$
x_{1}=\sum_{i=2}^{m(n)} \alpha_{i} x_{i}, \text { where } \alpha_{i}=-\lambda_{i} / \lambda_{1}, i=2, \ldots, m(n) .
$$

Let $0<\varepsilon<4$. We take $y_{i} \in \operatorname{span} C_{n}\left(a_{i}^{n}\right) \cap E$ such that

$$
\left\|y_{i}-x_{i}\right\|<(2 m(n) \alpha)^{-1} \varepsilon<\varepsilon / 2 \text { for every } i=1, \ldots, m(n),
$$

where $\alpha=\max \left\{\left[\left|\alpha_{i}\right|\right]+1: i=2, \ldots, m(n)\right\}$. (Recall that $[\alpha]$ denotes the greatest integer which is smaller than $\alpha$.) From Lemma 6 we get

$$
\text { supp } y_{i} \subset S_{i}^{n} \text { for every } i=1, \ldots, m(n) \text {. }
$$

Take $k \in \mathbb{N}$ such that $y_{i} \in E_{n+k}$ for every $i=1, \ldots, m(n)$. Let $z_{i}^{1}=\alpha_{i} y_{i}$ for $i=2, \ldots, m(n)$. Then we can denote

$$
z_{1}=y_{1}-\sum_{i=2}^{m(n)} \alpha_{i} y_{i}=y_{1}-\sum_{i=2}^{m(n)} z_{i}^{1} \in E_{n+k}
$$

whence

$$
y_{1}=z_{1}+\sum_{i=2}^{m(n)} z_{i}^{1}
$$

Note that

$$
\text { supp } y_{1} \subset S_{1}^{n} \text { and } \operatorname{supp} z_{i}^{1} \subset S_{i}^{n} \text { for } i=2, \ldots, m(n) \text {. }
$$


Then from (1) and (41) we have

$$
\begin{aligned}
\left\|z_{1}\right\| & =\left\|y_{1}-\sum_{i=2}^{m(n)} \alpha_{i} y_{i}\right\| \leq\left\|y_{1}-x_{1}\right\|+\left\|x_{1}-\sum_{i=2}^{m(n)} \alpha_{i} y_{i}\right\| \\
& <2^{-1} \varepsilon+\left\|\sum_{i=2}^{m(n)} \alpha_{i}\left(x_{i}-y_{i}\right)\right\| \leq 2^{-1} \varepsilon+\sum_{i=2}^{m(n)}\left\|\alpha_{i}\left(x_{i}-y_{i}\right)\right\| \\
& \leq 2^{-1} \varepsilon+\sum_{i=2}^{m(n)} \max \left\{\left[\alpha_{i}\right]+1: i=2, \ldots, m(n)\right\}\left\|x_{i}-y_{i}\right\| \\
& <2^{-1} \varepsilon+m(n) \alpha(2 m(n) \alpha)^{-1} \varepsilon=\varepsilon .
\end{aligned}
$$

Now, recall that $y_{1} \in E_{n+k}$. Our strategy is to "cut off" the "top" of $y_{1}$ step by step until we get an element in the space $E_{n}$.

The first step. In this step we cut a small element $u_{1}$ off from $y_{1}$ so that $y_{1}-u_{1} \in$ $E_{n+k-1}$.

Since $\left\|z_{1}\right\|<\varepsilon<4$, it follows from Theorem 4(iii) that there exists an $r \in \mathbb{N}$ such that $N_{r}\left(z_{1}\right)<4$. Replacing $r$ or $n+k$ by $\max \{r, n+k\}$ if necessary, we may assume that $r=n+k$. Hence from Theorem 4 (iv) we get $N_{n+k}\left(z_{1}\right)=\left\|z_{1}\right\|<\varepsilon$. Therefore by Theorem 4(ii) there exist

$$
\begin{gathered}
y_{i}^{n+k-1} \in E_{n+k}\left(S_{i}^{n+k-1}\right), i=1, \ldots, m(n+k-1), \text { and } z_{2} \in E_{n+k-1} \text { such that } \\
z_{1}=z_{2}+\sum_{i=1}^{m(n+k-1)} y_{i}^{n+k-1}
\end{gathered}
$$

and

$$
N_{n+k-1}\left(z_{2}\right)+\sum_{i=1}^{m(n+k-1)} N_{n+k}\left(S_{i}^{n+k-1}\right)\left(y_{i}^{n+k-1}\right)<\varepsilon .
$$

Denote

$$
\varepsilon_{1}=\sum_{i=1}^{m(n+k-1)} N_{n+k}\left(S_{i}^{n+k-1}\right)\left(y_{i}^{n+k-1}\right) .
$$

Then from (45) we have

$$
N_{n+k-1}\left(z_{2}\right)<\varepsilon-\varepsilon_{1}
$$

Let

$$
v_{i}=\sum_{j \in T(i)} y_{j}^{n+k-1}, \text { where } T(i)=\left\{j: S_{j}^{n+k-1} \subset S_{i}^{n}\right\} \text { for } i=1, \ldots, m(n) .
$$

Let $u_{1}=v_{1}$. Then we have $\operatorname{supp}\left(y_{1}-u_{1}\right) \subset S_{1}^{n}$. We prove:

Claim 7. $y_{1}-u_{1} \in E_{n+k-1}$. 
Proof. Observe that

$$
\begin{aligned}
z_{2} & =z_{1}-\sum_{i=1}^{m(n+k-1)} y_{i}^{n+k-1}=y_{1}-\sum_{i=2}^{m(n)} \alpha_{i} y_{i}-\sum_{i=1}^{m(n+k-1)} y_{i}^{n+k-1} \\
& =y_{1}-\sum_{i=2}^{m(n)} \alpha_{i} y_{i}-\sum_{i=1}^{m(n)} \sum_{j \in T(i)} y_{j}^{n+k-1}=y_{1}-\sum_{i=2}^{m(n)} \alpha_{i} y_{i}-\sum_{i=1}^{m(n)} v_{i} \\
& =y_{1}-v_{1}-\sum_{i=2}^{m(n)}\left(\alpha_{i} y_{i}+v_{i}\right)=-\sum_{i=1}^{m(n)}\left(\alpha_{i} y_{i}+v_{i}\right), \text { where } \alpha_{1}=-1 .
\end{aligned}
$$

Since $z_{2} \in E_{n+k-1}$, we can write $-z_{2}=\sum_{j=1}^{m(n+k-1)} w_{j}$, where

$$
w_{j}=\lambda_{j} m(n+k-1) \chi_{S_{j}^{n+k-1}}, \lambda_{j} \in \mathbb{R}, j=1, \ldots, m(n+k-1) .
$$

Denote $\varphi_{i}=\sum_{S_{j}^{n+k-1} \subset S_{i}^{n}} w_{j}$. Since

$$
\varphi_{i} \in E_{n+k-1} \text { and } \operatorname{supp} \varphi_{i} \subset S_{i}^{n} \text { for every } i=1, \ldots, m(n) \text {, }
$$

we get

$$
-z_{2}=\sum_{i=1}^{m(n)} \varphi_{i}=\sum_{i=1}^{m(n)}\left(\alpha_{i} y_{i}+v_{i}\right) .
$$

Therefore

$$
\sum_{i=1}^{m(n)}\left(\alpha_{i} y_{i}+v_{i}-\varphi_{i}\right)=\theta .
$$

From (42) and (48) we have

$$
\operatorname{supp}\left(\alpha_{i} y_{i}+v_{i}\right) \subset S_{i}^{n} \text { for every } i=1, \ldots, m(n) .
$$

Hence

$$
\operatorname{supp}\left(\alpha_{i} y_{i}+v_{i}-\varphi_{i}\right) \subset S_{i}^{n} \text { for every } i=1, \ldots, m(n) .
$$

Therefore from (49) we obtain

$$
\alpha_{i} y_{i}+v_{i}-\varphi_{i}=\theta \text { for } i=1, \ldots, m(n) .
$$

It follows that

$$
\alpha_{i} y_{i}+v_{i}=\varphi_{i} \in E_{n+k-1} \text { for } i=1, \ldots, m(n) .
$$

Since $\alpha_{1}=1$ we get

$$
\alpha_{1} y_{1}+v_{1}=-y_{1}+u_{1}=-\left(y_{1}-u_{1}\right) \in E_{n+k-1} .
$$

Consequently $y_{1}-u_{1} \in E_{n+k-1}$, and the claim is proved.

Remark 7. Denote $u_{i}^{1}=v_{i}$ and $z_{i}^{2}=z_{i}^{1}+v_{i}=z_{i}^{1}+u_{i}^{1}$ for $i=1, \ldots, m(n)$. Then from (44) and (48) we get

$$
y_{1}-u_{1}=z_{2}+\sum_{i=2}^{m(n)} z_{i}^{2} \in E_{n+k-1}
$$


moreover

$$
\operatorname{supp}\left(y_{1}-u_{1}\right) \subset S_{1}^{n} \text { and } \operatorname{supp} z_{i}^{2} \subset S_{i}^{n} \text { for } i=2, \ldots, m(n) .
$$

Therefore (43) and (44) hold true if $y_{1}, z_{i}^{1}$ are replaced by $y_{1}-u_{1}$ and $z_{i}^{2}$, respectively.

The induction step. We continue to cut a small element $u_{2}$ off from $y_{1}-u_{1}$ so that $y_{1}-u_{1}-u_{2} \in E_{n+k-2}$ and so on. In the $k$-th step we get

$$
y_{1}-u_{1}-u_{2}-\cdots-u_{k} \in E_{n} .
$$

This process is done by induction as follows: Since

$$
z_{2} \in E_{n+k-1} \text { and } N_{n+k-1}\left(z_{2}\right)<\varepsilon-\varepsilon_{1}<4,
$$

we can obtain $z_{3} \in E_{n+k-2}$ and $y_{i}^{n+k-2} \in E_{n+k-1}\left(S_{i}^{n+k-2}\right), i=1, \ldots, m(n+k-1)$, such that

$$
z_{2}=z_{3}+\sum_{i=1}^{m(n+k-2)} y_{i}^{n+k-2}
$$

and

$$
N_{n+k-2}\left(z_{3}\right)+\sum_{i=1}^{m(n+k-2)} N_{n+k-2}\left(S_{i}^{n+k-2}\right)\left(y_{i}^{n+k-2}\right)<\varepsilon-\varepsilon_{1} .
$$

Denote

$$
\begin{aligned}
T(2, i) & =\left\{t: S_{t}^{n+k-2} \subset S_{i}^{n}\right\}, i=1, \ldots, m(n) ; \\
u_{i}^{2} & =\sum_{t \in T(2, i)} y_{t}^{n+k-2} ; i=1, \ldots, m(n) .
\end{aligned}
$$

Notice $u_{2}=u_{1}^{2}$. Let $z_{i}^{3}=z_{i}^{2}+u_{i}^{2}$ for $i=2, \ldots, m(n)$. Then it is easy to see that $y_{1}-u_{1}, z_{2}, z_{i}^{2}$ and $E_{n+k-1}$ in Remark 7 can be replaced by $y_{1}-u_{1}-u_{2}, z_{3}, z_{i}^{3}$ and $E_{n+k-2}$ respectively.

Now, for every $j=1, \ldots, k$ and $i=1, \ldots, m(n+k-j)$ we choose $y_{i}^{n+k-j} \in$ $E_{n+k-j+1}\left(S_{i}^{n+k-j}\right)$ and $z_{j+1} \in E_{n+k-j}$ such that

$$
z_{j}=z_{j+1}+\sum_{i=1}^{m(n+k-j)} y_{i}^{n+k-j}
$$

and

$$
\begin{aligned}
& N_{n+k-j}\left(z_{j+1}\right)+\sum_{i=1}^{m(n+k-j)} N_{n+k-j+1}\left(S_{i}^{n+k-j}\right)\left(y_{i}^{n+k-j}\right) \\
& <\varepsilon-\sum_{t=1}^{j-1} \varepsilon_{t}
\end{aligned}
$$

where

$$
\varepsilon_{t}=\sum_{i=1}^{m(n+k-t)} N_{n+k-t+1}\left(S_{i}^{n+k-t}\right)\left(y_{i}^{n+k-t}\right)
$$


(Observe that $\varepsilon-\varepsilon_{1}>0$, by (47), and $\varepsilon-\sum_{t=1}^{j-1} \varepsilon_{t}>0$ by induction.) Then from (52) we have $N_{n+k-j}\left(z_{j+1}\right)<\varepsilon-\sum_{t=1}^{j} \varepsilon_{t}$. Observe that

$$
\sum_{j=1}^{k} \sum_{i=1}^{m(n+k-j)} N_{n+k-j+1}\left(S_{i}^{n+k-j}\right)\left(y_{i}^{n+k-j}\right)=\sum_{j=1}^{k} \varepsilon_{j}<\varepsilon .
$$

Denote

$$
\begin{aligned}
T(j, i) & =\left\{t: S_{t}^{n+k-j} \subset S_{i}^{n}\right\}, i=1, \ldots, m(n) ; \\
u_{i}^{j} & =\sum_{t \in T(j, i)} y_{t}^{n+k-j} .
\end{aligned}
$$

Notice $u_{j}=u_{1}^{j}$. Let $z_{i}^{j}=z_{i}^{j-1}+u_{i}^{j-1}$ for $i=2, \ldots, m(n)$. Then from Theorem 4 (ii)-(iv) we get

$$
\begin{aligned}
\left\|u_{j}\right\| & \leq \sum_{i \in T(j, 1)} N_{n+k-j+1}\left(S_{i}^{n+k-j}\right)\left(y_{i}^{n+k-j}\right) \\
& \leq \sum_{i=1}^{m(n+k-j)} N_{n+k-j+1}\left(S_{i}^{n+k-j}\right)\left(y_{i}^{n+k-j}\right)=\varepsilon_{j} .
\end{aligned}
$$

Let $z=\sum_{j=1}^{k} u_{j}$. Since $\left\{N_{n}(x)\right\}$ is a decreasing sequence for every $x \in E$, from (53) and (54) we have

$$
\|z\| \leq \sum_{j=1}^{k}\left\|u_{j}\right\| \leq \sum_{j=1}^{k} \varepsilon_{j}<\varepsilon .
$$

Then it is easy to see that $y_{1}-u_{1}, z_{2}, z_{i}^{2}$ and $E_{n+k-1}$ in Remark 7 can be replaced by $y_{1}-\sum_{j=1}^{k} u_{j}=y_{1}-z, z_{k+1}, \quad z_{i}^{k+1}$ and $E_{n+k-k}=E_{n}$ respectively. Therefore we get $y_{1}-z=y_{1}-\sum_{j=1}^{k} u_{j} \in E_{n}$. Observe that

$$
\left\|x_{1}-\left(y_{1}-z\right)\right\| \leq\left\|x_{1}-y_{1}\right\|+\|z\|<2 \varepsilon .
$$

Therefore $\left\|x_{1}-E_{n}\right\|<2 \varepsilon$. Since $\varepsilon$ is arbitrarily small we infer that $x_{1} \in E_{n}$. So, $x_{1} \in E_{n} \cap \operatorname{span} C_{n}\left(a_{1}^{n}\right)$. Hence from Lemma 6 we get

$$
x_{1}=t_{1} a_{1}^{n}=t_{1} m(n) \chi_{S_{1}^{n}} \text { for some } t_{1} \in \mathbb{R} .
$$

Thus we have shown that if $\lambda_{i} \neq 0$, then $x_{i}=t_{i} m(n) \chi_{S_{i}^{n}}$ for some $t_{i} \in \mathbb{R}$. Since $\left\{m(n) \chi_{S_{i}^{n}}: i=1, \ldots, m(n)\right\}$ is linearly independent, it follows from (40) that $t_{i}=0$, if $\lambda_{i} \neq 0$. This contradicts the fact that $x_{i} \in \operatorname{span} C_{n}\left(a_{i}^{n}\right) \backslash\{\theta\}$ for every $i=1, \ldots, m(n)$. Theorem 5 is proved.

From Theorem 5 and from $[\mathrm{CDM}]$ we obtain the following result which answers affirmatively a problem of Dobrowolski and Mogilski; see [DM], Question 5-5 (Question 575).

Corollary. For any dense $\sigma$-fd-compact convex subset $W$ of $C$, we have $(C, W) \simeq$ $\left(Q, Q^{f}\right)$, where $Q$ denotes the Hilbert cube, and

$$
Q^{f}=\left\{x=\left(x_{n}\right) \in Q: x_{n}=0 \text { for almost all } n \in \mathbb{N}\right\}
$$

and " $\sigma$-fd-compact" means a countable union of finite dimensional compact sets. 
Related to Theorem 5 we ask:

Question 1 [NT1]. Let $F$ denote the linear metric space constructed by Roberts [R1]:

(i) Is every convex subset of $F$ an AR?

(ii) Does every compact convex subset of $F$ have the fixed point property?

We do not know even if the whole space $F$ is an AR. It is of interest to know whether Theorem 2 still holds in the general case:

Question 2. Is the compact convex set $C$ defined by Theorem 1 an AR?

It should be noted that Condition (i) is essential in our proof of Theorem 2. However, as we observed in Remark 6, Condition (ii) can be dropped. By [NT2] $C$ has the fixed point property. So we may ask Question 2 in the following more general situation:

Question 3 [NT1]. Assume that $X$ is a compact convex set with the fixed point property. Is $X$ an AR?

\section{ACKNOWLEDGEMENTS}

The authors are grateful to the referee for his (her) comments.

\section{REFERENCES}

[BD] C. Bessaga and T. Dobrowolski, Some open problems on the border of infinite dimensional topology and functional analysis, Proceedings of the international conference on geometric topology, PWN, Warszawa 1980.

[CDM] D. Curtis, T. Dobrowolski and J. Mogilski, Some applications of the topological characterizations of the sigma-compact spaces $\ell_{f}^{2}$ nad $\Sigma$, Trans. Amer. Math. Soc. 284(1984), 837-847. MR 86i:54035

[DM] T. Dobrowolski and J. Mogilski, Problems on topological classification of incomplete metric spaces, Open problems in topology, J. van Mill and G. M. Reed (Editors) Elsevier Science Publishers B. V. North-Holand 1990. MR 92c:54001

[G] R. Geoghegan, Open problems in infinite dimensional topology, Topology Proceedings, 4(1979), 287-330. MR 82a:57015

[K1] V. Klee, Shrinkable neighbourhoods in Hausdorff linear spaces, Math. Ann. 141(1960), 281-285. MR 24:A1003

[K2] V. Klee, Leray-Schauder theory without local convexity, Math. Ann. 141(1960), 286-296. MR 24:A1004

[KM] M. G. Krein and D. P. Milman, On extreme points of regular convex sets, Studia Math. 9(1940), 133-138. MR 3:90a

[KP] N. J. Kalton and N. T. Peck, A re-examination of Roberts' example of a compact convex set with no extreme points, Math. Ann. 253(1980), 89-101. MR 82h:46055

[KPR] N. J. Kalton, N. T. Peck and J. W. Roberts, An F-space sampler, London Math. Soc. Lecture Note Series 89(1984). MR 87c:46002

[N1] Nguyen To Nhu, Investigating the ANR-property of metric spaces, Fund. Math. 124(1984), 243-254; Correction, Fund. Math. 141(1992), 297. MR 86d:54018, MR 93k:54042

[N2] Nguyen To Nhu, The finite dimensional approximation property and the AR-property in needle point spaces, J. London Math. Soc. (to appear).

[NS] Nguyen To Nhu and Katsuro Sakai, The compact neighborhood extension property and the local equi-connectedness, Proc. Amer. Math. Soc. 121(1994), 259-265. MR 94g:54009

[NT1] Nguyen To Nhu and Le Hoang Tri, Every needle point space contains a compact convex AR-set with no extreme points, Proc. Amer. Math. Soc. 120(1994), 1261-1265. MR 94f: 54038

[NT2] Nguyen To Nhu and Le Hoang Tri, No Roberts space is a counter-example to Schauder's conjecture, Topology, 33(1994), 371-378. MR 95h:46014 
[R1] J. W. Roberts, A compact convex set with no extreme points, Studia Math. 60(1977), 255-266. MR 57:10595

[R2] J. W. Roberts, Pathological compact convex sets in the spaces $L_{p}, 0 \leq p<1$, The Altgeld Book, University of Illinois, 1976.

[Re] S. Rolewicz, Metric linear spaces, PWN, Warszawa 1972; Second publication, PWN, Warszawa 1982. MR 55:10993; MR 88i:46004a; MR 88i:46004b

Institute of Mathematics, P.O. Box 631, Bo Ho, Hanoi, Vietnam

Current address: Department of Mathematical Sciences, New Mexico State University, Las Cruces, New Mexico 88003-8001

E-mail address: nnguyen@nmsu.edu

Departamento de Geometria y Topologia, Facultad de Matematicas, Universidad Complutense de Madrid, 28040 Madrid, Spain

E-mail address: sanjurjo@sungt1.mat.ucm.es

Department of Mathematics, University of Vinh, Nghe An, Vietnam 